Introduction: Although the recommended optimal treatment of glioblastoma multiforme (GBM) is adjuvant chemoradiotherapy, trials in GBM have excluded patients older than 70 years. In this study, we aimed to assess overall survival (OS) and prognostic factors in elderly patients $(\geq 70$ years) with newly diagnosed GBM treated with radiotherapy (RT) \pm concurrent/adjuvant temozolomide (TMZ).

Material and methods: Inclusion criteria were patients $\geq 70$ years, pre-RT Karnofsky performance status (KPS) $\geq 60$, and time between diagnosis and start of RT $\leq 2$ months. A total of $40 \mathrm{pa}$ tients aged $\geq 70$ years, 12 female and 28 male, treated between January 2004 and December 2012, were evaluated. Median age was 73.5 years (range, 70-83 years). The median RT dose was 60 Gy (range, 30-62 Gy). Twenty-one $(52.5 \%)$ received concurrent $\mathrm{TMZ}$, and of those 12 (30\%) went on to receive adjuvant TMZ.

Results: The median OS was 7 months (95\% Cl: 5.45-8.54). One- and twoyear OS for the whole cohort was 38\% and $16 \%$, respectively. Sex, type of surgery, tumor size, and RT dose did not significantly affect the OS. Presence of concurrent TMZ $(p<0.005)$ and presence of adjuvant TMZ $(p<0.001)$ were associated with longer OS in our cohort.

Conclusions: $R T \pm T M Z$ seems to be a well-tolerated treatment in patients $\geq 70$ years with GBM. Even though no superiority was found between conventional or hypofractionated RT regimens $(p=0.405)$, the addition of concurrent and adjuvant TMZ to RT increased the OS in our study.

Key words: glioblastoma, radiotherapy, elderly, temozolomide, prognostic factors.

Contemp Oncol (Pozn) 2016; 20 (3): 251-255 DOI: $10.5114 /$ wo.2016.61569

\section{Radiotherapy with or without temozolomide in elderly patients aged $\geq 70$ years with glioblastoma}

\author{
Evrim Metcalfe ${ }^{1}$, Ozden Karaoglanoglu², Emine Akyazici ${ }^{2}$
}

${ }^{1}$ Department of Radiation Oncology, Eskisehir Osmangazi University School of Medicine, Eskisehir, Turkey

${ }^{2}$ Department of Radiation Oncology, Samsun Education and Investigation Hospital, Eskisehir, Turkey

\section{Introduction}

Glioblastoma (GBM, WHO grade IV) is the most life-threatening primary central nervous system malignancy and represents approximately $20 \%$ of primary brain tumors diagnosed in adults. Given the median age of 65 years at GBM diagnosis, a large proportion of cases occur in the elderly population $[1,2]$. In the landmark Stupp trial, concurrent oral temozolomide (TMZ) with RT followed by adjuvant TMZ improved survival in patients with GBM compared to postoperative RT alone. This study, however, excluded patients older than 70 years [1].

The treatment for elderly patients affected by GBM is a challenge in geriatric oncology. The standard of care for elderly patients with GBM remains undefined. No randomized trials have compared chemoradiotherapy with other medical alternatives since it has been presumed that elderly patients do not tolerate aggressive therapy [3, 4]. Consequently, clinicians have struggled with management decisions for elderly GBM patients. Nonetheless, with increasing life expectancy and expectations of better quality of life, there is current interest in GBM in the elderly [4-6].

Therefore, in this retrospective cohort analysis we assessed feasibility and outcome in patients $\geq 70$ years treated with RT with or without concurrent and adjuvant TMZ.

\section{Material and methods}

A retrospective study was performed using patient records, treatment plans and diagnostic imaging for patients who received RT \pm concurrent and adjuvant TMZ. Patients treated with RT for GBM between January 2004 and December 2012 were reviewed. Inclusion criteria were: age $\geq 70$ years, proven GBM WHO grade IV histopathology, no prior RT to the head-and-neck region, no prior use of chemotherapy or radiosensitizers, pre-RT Karnofsky Performance Status (KPS) $\geq 60$, and time between diagnosis and start of RT $\leq 2$ months.

Patients were treated in the supine position with a 3-point thermoplastic mask. Between 2004 and 2006, RT delivery was based on conventional simulator planning and parallel opposed fields. After September 2006, a 3D-conformal RT approach was introduced, and planning CT scans were acquired with intravenous contrast using 3-5 mm slices. The gross tumor volume (GTV) was outlined as areas of contrast enhancement on T1-weighted images on MRI. The clinical target volume (CTV) was constructed to include the GTV. The median standard planned adjuvant dose was 60 Gy in 30 fractions, and the median palliative dose was 30 Gy in 10 fractions to the planning target volume (PTV). Treatment was delivered with cobalt-60 until 2006, and then with ${ }^{60} \mathrm{Co}$ or a linear accelerator. 
Patients were given TMZ at a dose of $75 \mathrm{mg} / \mathrm{m}^{2} /$ day concurrent with RT, beginning on the day of the first fraction of RT, including weekends and holidays and ending on the day of the last radiation fraction. This was followed by up to 6 cycles of adjuvant TMZ at the dose of 150-200 mg/ $\mathrm{m}^{2}$ daily for 5 days every 28 days, as per the EORTC/NCIC protocol. All patients received trimethoprim-sulfamethoxazole for prophylaxis against Pneumocystis pneumonia.

Response assessment routinely included clinical and neurological examination, and imaging. Biopsy was considered in the event of clinical or radiological suspicion of progression. Patients were followed up with physical examination every $6-8$ weeks in the first year after treatment, every 3 months for an additional 2 years, and every 6 monthly until death.

Statistical analysis was performed using Statistical Package for Social Sciences software, version 16 (SPSS, Chicago, IL). OS was analyzed using the Kaplan-Meier method. A univariate analysis using Cox proportional regression analysis was used to examine the impact of multiple prognostic factors on OS. Factors identified with a $p$ value of $\leq 0.05$ on univariate analysis were then analyzed within a multivariate Cox model. All reported $p$ values are two-sided and differences were considered statistically significant when $p$ was $<0.05$.

\section{Results}

Forty elderly patients with GBM, 12 female and 28 male, with a median age of 73.5 years (range, 70-83 years), and a median KPS of 70 (range, 60-90) were treated at the Samsun Education and Investigation Hospital Department of Radiation Oncology between January 2004 and December 2012 using adjuvant RT \pm oral TMZ chemotherapy. Five (12.5\%) patients were alive at the median follow-up of 9 (2-43) months. Eleven (27.5\%) patients underwent gross total excision. The number of patients with pre-RT KPS = 60 was 13 (32.5\%). Patient characteristics

Table 1. Patient characteristics $(n=40)$

$\begin{array}{lcc}\text { Variables } & \text { No. } & \% \\ \text { Age at diagnosis - years } & & \\ \text { Median } & 74 & \\ \text { Range } & 70-83 & \\ \text { Sex - no. } & & \\ \text { Male } & 28 & 70 \\ \text { Female } & 12 & 30 \\ \text { KPS - no. } & & \\ 60 & 13 & 32.5 \\ >60 & 27 & 67.5 \\ \text { Type of surgery - no. } & & \\ \text { Gross total resection } & 11 & 27.5 \\ \text { Subtotal resection } & 26 & 65 \\ \text { Biopsy } & 3 & 7.5 \\ \text { Corticosteroid therapy - no. } & & \\ \text { Yes } & & \\ \text { No } & 32 & \\ \end{array}$

are summarized in Table 1. Twenty-eight (70\%) patients completed $\geq 60$ Gy of RT. Twenty-one (52.5\%) patients received concurrent TMZ; of these, 12 (30\%) patients received adjuvant TMZ. The median duration of RT was 42 days (range, 13-50 days), and the median RT dose was 60 Gy (range, 30-62 Gy) delivered in a median of 30 fractions (range, 10-31 fractions). RT and CT characteristics are summarized in Table 2.

Nineteen (47.5\%) patients were treated with RT alone. Twenty-four (60\%) patients completed their RT scheme without any interruption. The median number of adjuvant TMZ cycles was 3 (range, 0-6). One patient who was intended to receive $30 \mathrm{~Gy}$ in 10 fractions did not finish radiation therapy because of clinical tumor progression. In this case, death occurred after 24 Gy. Median OS was 7 months (95\% Cl: 5.45-8.54). One and two year OS for the whole cohort was $38 \%$ and $16 \%$, respectively. Overall, 35 of 40 patients died (87.5\%) with disease progression.

In univariate analysis, no significant difference was found in OS between conventional and hypofractionated RT schedules $\geq 60$ Gy vs. < 60 Gy, $p=0.405$ ) (Table 3). Younger age (70-75 years vs. $\geq 75$ years, MS: 9 months vs. 5 months, $p=0.03$ ), presence of concurrent TMZ (yes vs. no, MS: 11.7 months vs. 5 months, $p<0.005$ ), and adjuvant TMZ (yes vs. no, MS: 13 months vs. 5 months, $p<0.001$ ) were associated with longer OS in Kaplan Meier univariate analysis (Fig. 1). Adjuvant TMZ following concurrent RT+TMZ was found to be a significant predictor of OS on multivariate analysis (exp $=3.758,95 \% \mathrm{Cl}: 1.506-9.376$, $p=0.005)$. Univariate and multivariate analyses are summarized in Table 3.

Table 2. Radiotherapy (RT) and chemotherapy characteristics $(n=40)$

\begin{tabular}{|c|c|c|}
\hline Variables & No. & $\%$ \\
\hline \multicolumn{3}{|c|}{ Received $>90 \%$ of planned RT dose - no. } \\
\hline Yes & 28 & 70 \\
\hline No & 12 & 30 \\
\hline \multicolumn{3}{|c|}{ RT dose (Gray) } \\
\hline Median & 60 & \\
\hline Range & $24-62$ & \\
\hline \multicolumn{3}{|c|}{ Fraction size (Gray) } \\
\hline Median & 2 & \\
\hline Range & $1.8-3$ & \\
\hline \multicolumn{3}{|c|}{ Number of fractions } \\
\hline Median & 30 & \\
\hline Range & $8-31$ & \\
\hline \multicolumn{3}{|c|}{ Interruption or delay in RT (No.) } \\
\hline Yes & 16 & 40 \\
\hline No & 24 & 60 \\
\hline \multicolumn{3}{|c|}{ Concomitant TMZ } \\
\hline Yes & 21 & 52.5 \\
\hline No & 19 & 47.5 \\
\hline \multicolumn{3}{|c|}{ Adjuvant TMZ } \\
\hline Yes & 12 & 30 \\
\hline No & 28 & 70 \\
\hline
\end{tabular}



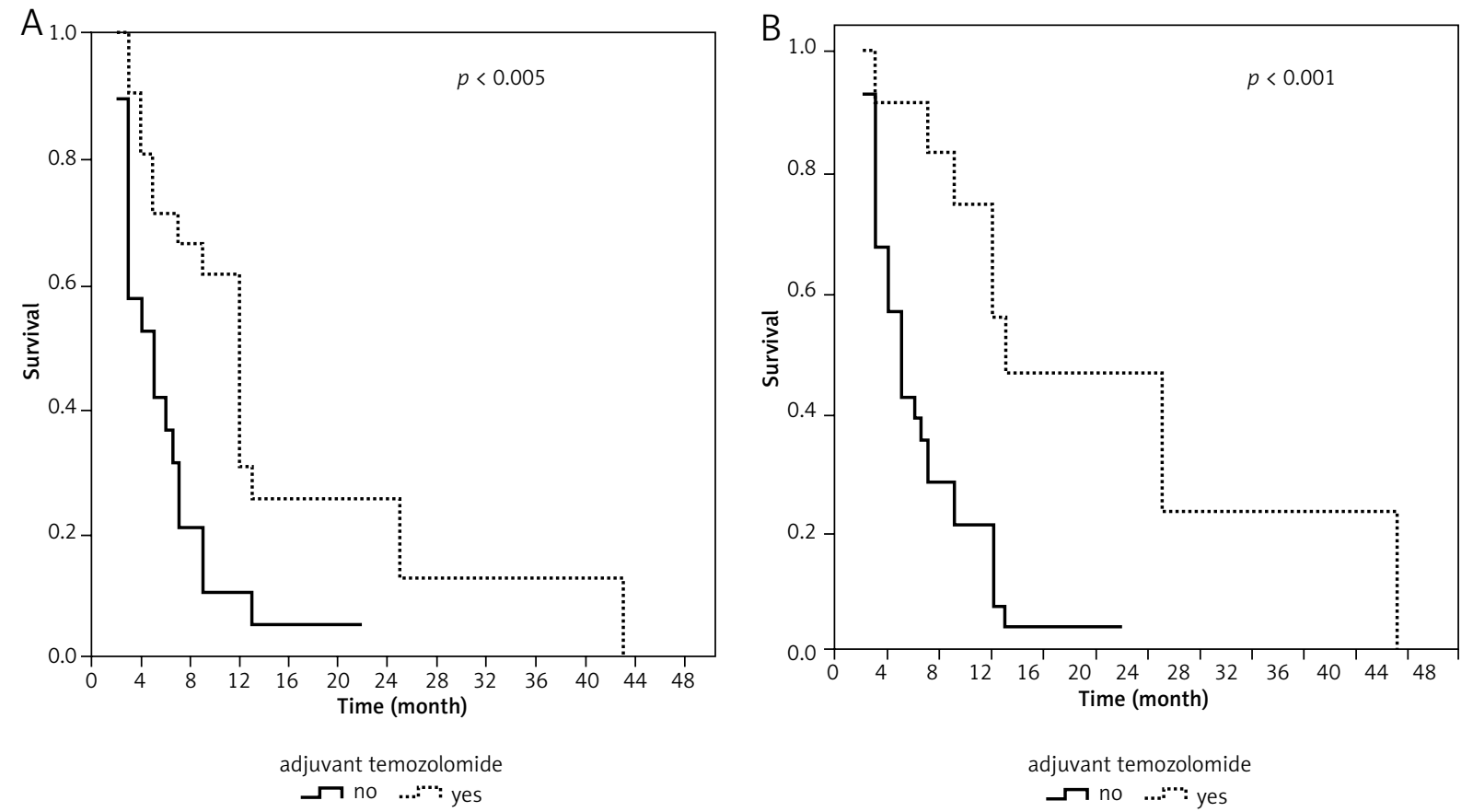

Fig. 1. Kaplan-Meier plots are shown relationship between overall survival (OS) and temozolomide (TMZ) in elderly patients with glioblastoma multiforme (GBM): A) Kaplan-Meier plot comparing OS in only radiotherapy (RT) vs. RT plus concurrent TMZ groups ( $p<0.005)$. B) Kaplan-Meier plot comparing OS in adjuvant TMZ vs. follow-up after RT+TMZ groups $(p<0.001)$

Table 3. Univariate and multivariate analysis of prognostic factors for overall survival (OS)

\begin{tabular}{|c|c|c|c|c|}
\hline \multirow[t]{2}{*}{ Variable } & \multicolumn{4}{|c|}{ Univariate (Kaplan Meier) } \\
\hline & OS (week) & $95 \% \mathrm{Cl}^{1}$ & $\mathrm{SE}^{2}$ & $p$ value \\
\hline \multicolumn{5}{|l|}{$\mathrm{KPS}^{3}$} \\
\hline 60 & 7.6 & $5.3-9.9$ & 1.18 & \\
\hline$>60$ & 8.8 & $6.2-11.4$ & 1.31 & 0.648 \\
\hline \multicolumn{5}{|l|}{ Extent of resection } \\
\hline Gross total resection & 8.1 & $4.1-12.2$ & 2.08 & \\
\hline Subtotal resection & 9.1 & $6.6-11.7$ & 1.30 & \\
\hline Biopsy & 5.5 & $4.1-6.9$ & 0.70 & 0.407 \\
\hline \multicolumn{5}{|l|}{ RT dose groups } \\
\hline$\geq 60$ Gy & 9.2 & $6.6-11.9$ & 1.37 & \\
\hline$<60 \mathrm{~Gy}$ & 7.2 & $4.6-9.8$ & 1.93 & 0.405 \\
\hline \multicolumn{5}{|l|}{ Concurrent $\mathrm{TMZ}^{4}$} \\
\hline Yes & 11.7 & $8.7-14.7$ & 1.56 & \\
\hline No & 5.1 & $3.8-6.4$ & 0.67 & $<0.001$ \\
\hline \multicolumn{5}{|l|}{ Adjuvant TMZ } \\
\hline Yes & 14.9 & $10.6-19.2$ & 2.21 & \\
\hline No & 5.9 & $4.6-7.2$ & 0.66 & $<0.001$ \\
\hline \multirow[t]{2}{*}{ Variable } & \multicolumn{4}{|c|}{ Multivariate (Cox regression) } \\
\hline & $\operatorname{Exp} \beta(\mathrm{SE})$ & $95 \% \mathrm{Cl}^{1}$ for $\exp \beta$ & $\mathrm{SE}^{2}$ & $p$ value \\
\hline Adjuvant TMZ & 3.758 & $1.506-9.376$ & $\left(0.466^{\star}\right)$ & 0.005 \\
\hline YES Vs. NO & & & & \\
\hline $\begin{array}{l}\text { Confidence interval } \\
\text { Standard error } \\
\text { Karnofsky performance status } \\
\text { Temozolomide }\end{array}$ & & & & \\
\hline
\end{tabular}




\section{Discussion}

This study shows that addition of adjuvant TMZ followed by concurrent TMZ with either short course (<60 Gy) or long course ( $\geq 60 \mathrm{~Gy}$ ) RT prolongs overall survival in patients with newly diagnosed GBM who are 70 years of age or older. OS in this small cohort of patients was comparable to patients treated with current standard of care therapy.

The optimal management of GBM in the elderly remains an area of controversy. Most studies demonstrate OS increases of several months among elderly GBM patients who receive surgery, $\mathrm{RT}$, and/or $\mathrm{TMZ}$. It is not clear whether the increased survival reflects treatment efficacy or better patient performance status. The NCCN guidelines currently include a category $2 \mathrm{~B}$ recommendation for combined RT and TMZ in patients with GBM aged > 70. Previously published non-randomized data suggest that either TMZ or RT alone results in similar overall survival rates in elderly patients with GBM. Although there is concern that chemotherapy causes excess toxicity and is less efficacious in this population, a previous phase II study published by Chinot et al. showed that TMZ is a well-tolerated oral chemotherapeutic agent. This study evaluated patients with GBM aged > 70 years treated with TMZ alone as first line therapy, and reported a median overall survival of 6.4 months. Based on these data, in subsequent studies, the authors treated elderly GBM patients with $T M Z$ alone or with RT[7-9].

The other area of contention for elderly patients with GBM is RT fractionation and total dose. Data from several prospective but nonrandomized studies have suggested that survival in patients receiving shorter-course RT is similar to that of historical controls treated conventionally. In the study by Roa et al., 100 patients with GBM aged > 60 years were randomized to receive either hypofractionated RT (40 Gy in 3 weeks) or standard dose RT (60 Gy in 6 weeks). The authors observed no significant difference in OS between the two study groups [10]. In another study by Keime-Guibert et al., 85 patients with GBM aged 70 years or older were randomized to receive either RT (50 Gy in 6 weeks) or supportive care alone [11]. This trial was closed early after an interim analysis showed a significant OS improvement in the RT group (HR: 0.47; 29.1 weeks vs. 16.9 weeks, $p=0.002$ ). There were no severe toxicities related to RT, and quality of life did not differ significantly between the treatment groups [11]. Brandes et al. evaluated patients with GBM aged > 65 years treated with highdose radiotherapy (60 Gy in 6 weeks) with concomitant TMZ. They reported a median OS of 13.7 months [12].

Results from our cohort showed that conventional and hypofractionated RT schemes were comparable in terms of OS ( $\geq 50$ Gy vs. < 50 Gy, $p=0.158$; or $\geq 60$ Gy vs. 30-60 Gy vs. $\leq 30 \mathrm{~Gy}, p=0.405)$. In another recent study, patients aged 70 years and older with GBM and KPS $\geq 70$ were randomized between $\mathrm{RT}$ in 1.8 Gy daily fractions to a total dose of 50 Gy plus supportive care, or supportive care alone. Analysis of preliminary results demonstrated the efficacy and safety of this hypofractionated accelerated regimen, which compared favorably with classically fractionated treatments [13]. Hypofractionated RT offers the advantage of a shorter treatment period, which is important in this group of patients with limited life expectancy [12-15].

Gzell et al. determined the outcome of 109 elderly patients with GBM with hypofractionated (40 Gy) or longcourse (60 Gy) external beam RT. Patients aged $>60$ years diagnosed with GBM managed with RT were identified as to ECOG performance status, RT dose and use of TMZ. Median OS of patients was 12 months, with age distribution from 61 to 88 years $(63 \%, 65-75)$. For age groups $65-75$ and $>75$, the survival was 12 and 9 months, respectively $(p=0.001)$. They found an association between longer survival and the use of 60 Gy (15 vs. 9 months, $p<0.0001$ ), and use of TMZ (13 vs. 7 months, $p<0.0001)$. They concluded that improved OS is possible in the elderly population when TMZ is added to RT. Those in the age group 65-75 may benefit from long-course RT with TMZ [16]. Similarly to this study, we found superiority of concurrent and adjuvant TMZ usage on OS in our patients with median age of 73.5 years (range, 70-83), even though there was no significant difference for OS between RT schemes.

Several studies have investigated the treatment patterns and outcomes among elderly GBM patients over the last 20-30 years. In the current literature, age remains one of the most powerful prognostic factors among GBM patients. More than $50 \%$ of patients with GBM are aged 65 years or older at the time of diagnosis, and the incidence rate of GBM in patients aged over 65 years is increasing rapidly. The standard of care for elderly GBM patients remains controversial. Although the historical standard for GBM management has been surgery followed by adjuvant RT, many studies have recommended concurrent and adjuvant chemotherapy. Assessment of RT duration and toxicities is increasingly popular among researchers, and longer treatment schemes may be considered onerous in many clinics if not associated with significantly prolonged survival. The landscape of treatment options for elderly GBM patients has changed substantially over the past decade and even in the past 2 years. Based on the available evidence, Arvold and Reardon have outlined suggested management options based on patient age and KPS. Thus, as treatment decisions are made regarding elderly patients with GBM, honest discussions between physicians and patients and their families are crucial in terms of survival outcomes and quality of life and patient preferences [17].

In conclusion, our experience in this retrospective study with a small number of elderly patients with GBM will need to be considered in the planning of future studies. TMZ appears to be an effective treatment concurrently with and following RT for elderly patients with newly diagnosed GBM. The RT scheme for elderly patients requires careful consideration, and future randomized multicenter trials will be necessary to evaluate the role of hypofractionated RT with TMZ in the treatment of patients $\geq 70$ years with newly diagnosed GBM. 
The authors would like to thank Dr. Louise Murray, St. James's Institute of Oncology, Leeds, UK for her contributions to this study.

The authors declare no conflict of interest.

\section{References}

1. Stark AM, Stepper W, Mehdorn HM. Outcome evaluation in glioblastoma patients using different ranking scores: KPS, GOS, mRS and MRC. Eur J Cancer Care (Engl) 2010; 19: 39-44.

2. Stupp R, Mason WP, van den Bent MJ, et al. Radiotherapy plus concomitant and adjuvant temozolomide for glioblastoma. N Engl Med 2005; 352: 987-96.

3. Oh SW, Jee TK, Kong DS, Nam DH, Lee JI, Seol HJ. Outcome of conventional treatment and prognostic factor in elderly glioblastoma patients. Acta Neurochir (Wien) 2014; 156: 641-51.

4. Niyazi M, Schwarz SB, Suchorska B, Belka C. Radiotherapy with and without temozolomide in elderly patients with glioblastoma. Strahlenther Onkol 2012; 188: 154-9.

5. Fiorentino A, De Bonis P, Chiesa S, Balducci M, Fusco V. Elderly patients with glioblastoma: the treatment challenge. Expert Rev Neurother 2013; 13: 1099-105.

6. Fiorentino A, Balducci M, De Bonis P, et al. Can elderly patients with newly diagnosed glioblastoma be enrolled in radiochemotherapy trials? Am J Clin Oncol 2015; 38: 23-7.

7. Chinot OL, Barrie M, Frauger E, et al. Phase II study of temozolomide without radiotherapy in newly diagnosed glioblastoma multiforme in an elderly populations. Cancer 2004; 100: 2208-14.

8. Gerstein J, Franz K, Steinbach JP, et al. Postoperative radiotherapy and concomitant temozolomide for elderly patients with glioblastoma. Radiother Oncol 2010; 97: 382-6.

9. Gerstein J, Franz K, Steinbach JP, Seifert V, Rödel C, Weiss C. Radiochemotherapy with temozolomide for patients with glioblastoma. Prognostic factors and long-term outcome of unselected patients from a single institution. Strahlenther Onkol 2011; 187: 722-8.

10. Roa W, Brasher PM, Bauman G, et al. Abbreviated course of ra diation therapy in older patients with glioblastoma multiforme: a prospective randomized clinical trial. J Clin Oncol 2004; 22: 1583-8.

11. Keime-Guibert F, Chinot O, Taillandier L, et al. Radiotherapy for glioblastoma in the elderly. N Engl J Med 2007; 356: 1527-35.

12. Brandes AA, Tosoni A, Franceschi E, et al. Temozolomide concomitant and adjuvant to radiotherapy in elderly patients with glio blastoma: correlation with MGMT promoter methylation status. Cancer 2009; 115: 3512-8.

13. Lopez S, Taillibert S, Idbaih A, Simon JM, Mazeron JJ. Should elderly patients with glioblastoma be proposed to radiotherapy? Cancer Radiother 2008; 12: 827-30

14. Jastaniyah N, Murtha A, Pervez N, et al. Phase I study of hypofractionated intensity modulated radiation therapy with concurrent and adjuvant temozolomide in patients with glioblastoma multiforme. Radiat Oncol 2013; 20: 38.

15. Reddy K, Damek D, Gaspar LE, et al. Phase II trial of hypofractionated IMRT with temozolomide for patients with newly diagnosed glioblastoma multiforme. Int J Radiat Oncol Biol Phys 2012; 84 655-60.

16. Gzell C, Wheeler H, Guo L, Kastelan M, Back M. Elderly patients aged 65-75 years with glioblastoma multiforme may benefit from long course radiation therapy with temozolomide. J Neurooncol 2014; 119: 187-96

17. Arvold ND, Reardon DA. Treatment options and outcomes for glio blastoma in the elderly patient. Clin Interv Aging 2014; 9: 357-67.

\section{Address for correspondence}

\section{Evrim Metcalfe}

Eskisehir Osmangazi University

School of Medicine

Department of Radiation Oncology

Odunpazari 26480, Eskisehir, Turkey

e-mail: evrimbayman@hotmail.com

Submitted: 3.09 .2014

Accepted: $\quad 20.07 .2015$ 